\title{
Non-local thermodynamic equilibrium inversions from a 3D magnetohydrodynamic chromospheric model
}

\author{
J. de la Cruz Rodríguez ${ }^{1,2,3}$, H. Socas-Navarro ${ }^{4}$, M. Carlsson ${ }^{3,5}$, and J. Leenaarts ${ }^{3,6}$ \\ 1 Department of Physics and Astronomy, Uppsala University, Box 516, 75120 Uppsala, Sweden \\ e-mail: jaime.cruz@physics.uu.se \\ 2 Institute for Solar Physics of the Royal Swedish Academy of Sciences, AlbaNova, 10691 Stockholm, Sweden \\ 3 Institute of Theoretical Astrophysics, University of Oslo, PO Box 1029 Blindern, 0315 Oslo, Norway \\ 4 Instituto de Astrofísica de Canarias, Avda Vía Láctea S/N, 38205 La Laguna, Tenerife, Spain \\ 5 Center of Mathematics for Applications, University of Oslo, PO Box 1053 Blindern, 0316 Oslo, Norway \\ ${ }^{6}$ Utrecht University, PO Box 800003508 TA Utrecht, The Netherlands
}

Received 16 January 2012 / Accepted 26 April 2012

\begin{abstract}
Context. The structure of the solar chromosphere is believed to be governed by magnetic fields, even in quiet-Sun regions that have a relatively weak photospheric field. During the past decade inversion methods have emerged as powerful tools for analyzing the chromosphere of active regions. The applicability of inversions to infer the stratification of the physical conditions in a dynamic 3D solar chromosphere has not yet been studied in detail.

Aims. This study aims to establish the diagnostic capabilities of non-local thermodynamical equilibrium (NLTE) inversion techniques of Stokes profiles induced by the Zeeman effect in the Ca II $\lambda 8542 \AA$ line.

Methods. We computed the Ca II atomic level populations in a snapshot from a 3D radiation-MHD simulation of the quiet solar atmosphere in non-LTE using the 3D radiative transfer code Multi3d. These populations were used to compute synthetic full-Stokes profiles in the Ca II $\lambda 8542 \AA$ line using $1.5 \mathrm{D}$ radiative transfer and the inversion code Nicole. The profiles were then spectrally degraded to account for finite filter width, and Gaussian noise was added to account for finite photon flux. These profiles were inverted using Nicole and the results were compared with the original model atmosphere.

Results. Our NLTE inversions applied to quiet-Sun synthetic observations provide reasonably good estimates of the chromospheric magnetic field, line-of-sight velocities and somewhat less accurate, but still very useful, estimates of the temperature. Threedimensional scattering of photons cause cool pockets in the chromosphere to be invisible in the line profile and consequently they are also not recovered by the inversions. To successfully detect Stokes linear polarization in this quiet snapshot, a noise level below $10^{-3.5}$ is necessary.
\end{abstract}

Key words. Sun: chromosphere - Sun: magnetic topology - radiative transfer - polarization - magnetohydrodynamics (MHD)

\section{Introduction}

Measuring the magnetic field of the solar chromosphere is an outstanding challenge for observers. The reason for this is threefold: there are not many lines available in the optical spectrum with a sufficiently high opacity to place the formation in the chromosphere, the polarimetric response is limited, and the few lines available have a non-local component to their formation (Socas-Navarro \& Trujillo Bueno 1997; Pietarila et al. 2006; Manso Sainz \& Trujillo Bueno 2010; Leenaarts et al. 2012).

The situation is much simpler in the photosphere: to model most Fe I photospheric lines, it is customary to assume local thermodynamic equilibrium (LTE, see Rutten \& Kostik 1982), which means that the atom population densities can be computed directly from the local temperature and electron density at each point. Unfortunately, most chromospheric lines require a non-LTE (NLTE hereafter) treatment of the radiative transfer problem.

Out of the available chromospheric diagnostic lines, the Ca II infrared (IR) triplet $(\lambda 8498, \lambda 8542, \lambda 8662)$ constitutes a good compromise of modeling efforts, polarimetric sensitivity and observational requirements; partial redistribution effects are negligible (Uitenbroek 1989) and almost the entire calcium is singly ionized under typical solar chromospheric conditions such that time-dependent ionization effects are negligible and the statistical equilibrium equations can be used to compute the level populations (Wedemeyer-Böhm \& Carlsson 2011).

The Ca II IR triplet lines have relatively low effective Landé factors $\left(g_{8498}=1.07, g_{8542}=1.10, g_{8662}=0.87\right)$ and broad Gaussian line cores compared to photospheric lines. This results in low polarization signals compared to those from photospheric lines. However, the triplet lines have the advantage that they can be observed over the entire solar disk (as opposed to the He I $\lambda 10830$ multiplet, which is visible only in active patches) and provide information also on the plasma temperature.

The development of inversion techniques has improved our capabilities to infer physical quantities from the chromosphere (see Trujillo Bueno 2010; Asensio Ramos et al. 2012, and references therein). The first full-Stokes NLTE inversions of the $\mathrm{Ca}$ II infrared lines were carried out by Socas-Navarro et al. (2000). Their approach is ideal for analyzing active region and network observations where the magnetic field is strong. To analyze quiet-Sun observations, very high sensitivity is required, especially in Stokes $Q$ and $U$, where signals are expected to be very low. 
This study aims to improve our understanding of chromospheric magnetic field diagnostics. To this end we will use 3D simulations to study NLTE inversions of quiet-Sun observations to quantify their reliability. We will also study the detectability of Zeeman-induced polarization; instrumental limitations and requirements. The goal is to investigate the polarimetric properties of the $\mathrm{Ca}$ II IR lines and the regime in which inversions yield realistic results.

A 3D magnetohydrodynamic (MHD) simulation is used to compute synthetic full-Stokes profiles with a realistic 3D solution of the NLTE problem. The resulting synthetic profiles are inverted assuming plane-parallel geometry on a pixel-by-pixel basis. Realistic values of noise and instrumental degradation are applied to the simulated observations before carrying out the inversions.

In real observations, Stokes $Q$ and $U$ are produced by the combined action of scattering polarization and transverse Zeeman effect when $10 \mathrm{G}<B<100 \mathrm{G}$ (Manso Sainz \& Trujillo Bueno 2010). Within the mentioned range, Hanle signals are expected to have $Q$ and $U$ amplitudes $\leq 10^{-4}$ at solar disk center and $\leq 10^{-3}$ close to the limb. In active regions the Zeeman contribution would be dominant. We emphasize that this study only accounts for Zeeman-induced polarization, a simplification that is needed given the computational demands of the problem (see Carlin et al. 2012).

The $3 \mathrm{D}$ simulation, the radiative transfer calculations and the simulated observations are explained in Sect. 2, the detectability of the Stokes signal is discussed in Sect. 3 and the inversion code and the inversion results are discussed in Sect. 4. The conclusions are given in Sect. 5.

\section{Simulated observations}

\subsection{D MHD model}

We used a snapshot from a 3D radiation-MHD simulation of the quiet Sun that extends from the upper convection zone to the low corona. The simulation was carried out with the Oslo stagger code (OSC, Hansteen et al. 2007) which includes nongray radiative losses using multi-group opacities with scattering, NLTE radiative losses in the chromosphere, optically thin radiative losses in the corona and thermal conduction along the magnetic field lines. The simulation domain consists of a grid of $256 \times 128 \times 160$ points covering a physical range of $16.6 \times 8.3 \times 15.5 \mathrm{Mm}$, extending from the upper convection zone to the lower corona (from 1.5 Mm below to $14 \mathrm{Mm}$ above average optical depth unity at $5000 \AA$ ). The simulation has an average magnetic field strength of $150 \mathrm{G}$ in the photosphere, a value consistent with the mean field strength inferred by Trujillo Bueno et al. (2004) via the Hanle effect. The magnetic field is mostly concentrated in intergranular lanes at photospheric heights, whereas in the chromosphere it tends to fill up more space. At $z=1000 \mathrm{~km}$, the average magnetic field strength is $79 \mathrm{G}$.

Figure 1 illustrates the vertical component of $\boldsymbol{B}$ in the photosphere (bottom) and in the low chromosphere (top). The simulation snapshot used in this work is the same as in Leenaarts et al. (2009, 2010) and Rutten et al. (2011).

\subsection{Radiative transfer}

Our synthetic full Stokes profiles are computed in two steps. First, population densities are calculated with Multi3D (Leenaarts \& Carlsson 2009), which allows one to evaluate

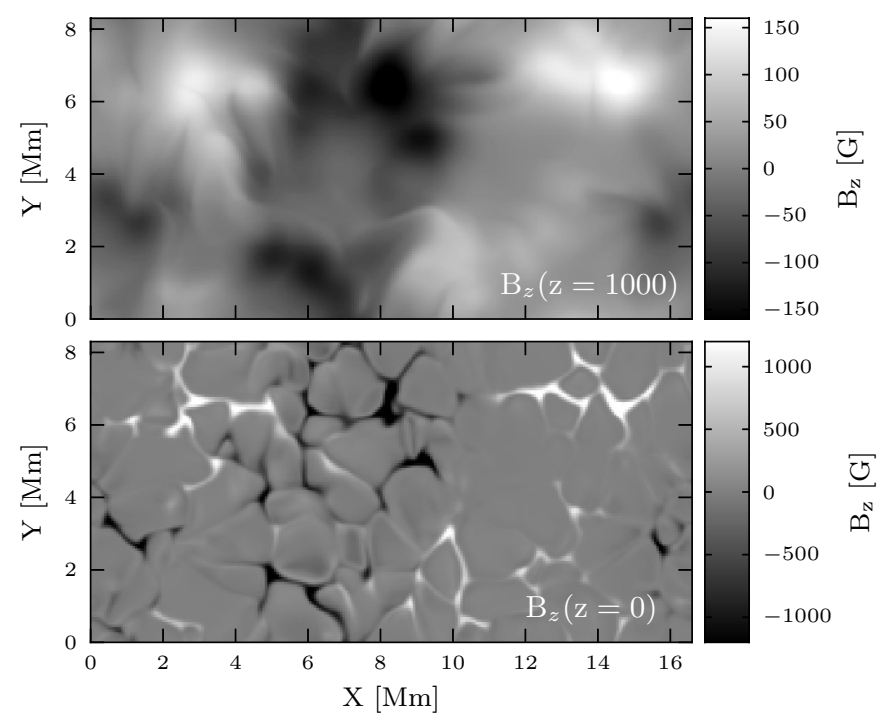

Fig. 1. Vertical component of the magnetic field, $B_{z}$, at horizontal slices through the 3D model atmosphere at $z=1000 \mathrm{~km}$ (top) and $z=0 \mathrm{~km}$ (bottom).

the $3 \mathrm{D}$ radiation field (3D NLTE). The Ca II atom model used in this work consists of five bound levels plus a continuum. We calculate the departure coefficients, defined as

$b_{i}=\frac{n_{i}}{n_{i}^{*}}$,

where $n_{i}$ is the population density of an atomic level $i$ computed in NLTE and $n_{i}^{*}$ is the equivalent LTE population density.

In the second step, the same atmospheric model is used to compute the LTE solution with the non-LTE inversion code based on the Lorien engine (Nicole, Socas-Navarro et al., in prep.). The departure coefficients obtained previously with Multi3D are applied to the LTE populations. Then, each column is resampled to a grid that is optimized for the radiative transfer computations in Ca II lines: for each column the model is truncated at the height where the temperature is higher than $50000 \mathrm{~K}$. The grid points are then distributed according to gradients in temperature, density and opacity, placing more points where the gradients are steep. The temperature, electron density, mass density, velocity, and departure coefficients are interpolated to the new grid using an accurate interpolation algorithm based on Hermitian splines (Hill 1982).

Leenaarts et al. (2009) pointed out that the Gaussian core of the synthetic Ca II $\lambda 8542 \AA$ Stokes I profile is narrower and steeper than those from observations, probably from the lack of small scale random motions in the model. Assuming Zeeman splitting in a weak-field regime, the shape of Stokes $Q$ and $V$ can be expressed as a function of the first derivative of the intensity (Landi Degl'Innocenti \& Landolfi 2004).

$$
\begin{aligned}
& Q(\lambda)=-\frac{1}{4} \Delta \lambda_{B}^{2} \bar{G} \frac{\eta^{\prime \prime}}{\eta^{\prime}}\left(\frac{\partial I}{\partial \lambda}\right) \\
& V(\lambda)=-\Delta \lambda_{B} \bar{g} \cos \theta\left(\frac{\partial I}{\partial \lambda}\right),
\end{aligned}
$$

where $\Delta \lambda_{B}$ represents the Zeeman splitting of the line, $\bar{g}$ is the Landé factor, $\theta$ is the inclination, $\bar{G}$ depends on the quantum numbers of the line and $\eta$ is the Voigt profile. According to Eqs. (2) and (3) the amplitude of Stokes $Q$ and $V$ is enhanced 


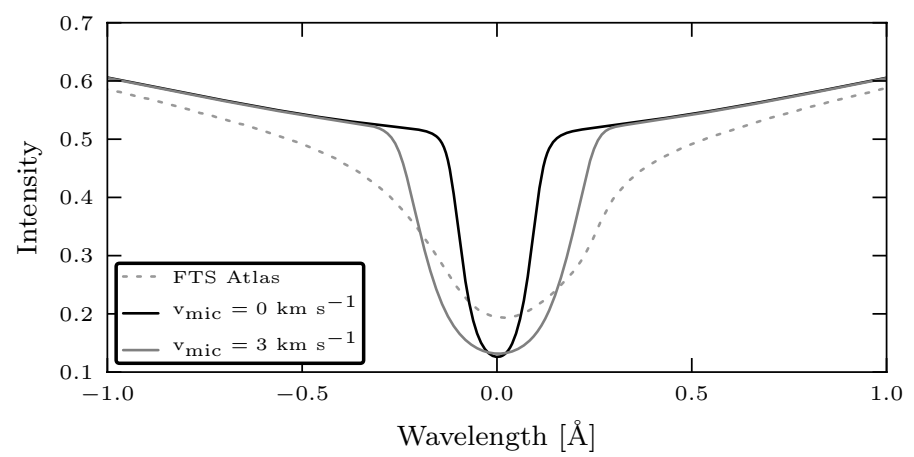

Fig. 2. Spatially-averaged profile without micro-turbulence (blacksolid), with micro-turbulence (gray-solid) and the FTS atlas (gray-dashed).

if the slope of Stokes $I$ is steep. Thus, steeper Stokes $I$ profiles produce more strongly peaked Stokes $Q, U$ and $V$ profiles. Therefore, our Stokes $Q, U$ and $V$ spectra would be unrealistically strong and narrow, which would lead to an overestimation of the effect of spectral smearing and an underestimation of the effect of photon noise.

To make more realistic predictions, we introduced an artificial micro-turbulence in the model. Without micro-turbulence, the full width at half maximum (FWHM) of the chromospheric core, measured in our spatially resolved spectra is about $180 \mathrm{~m} \AA$. Corresponding measurements from quiet-Sun line profiles were carried out by Cauzzi et al. (2009), who found values in the range 450-550 m $\AA$. This means that our profiles are of the order of a factor 2.5 narrower than observed, assuming that instrumental degradation has a negligible effect on the FWHM of the profiles. By artificially introducing micro-turbulence of $3 \mathrm{~km} \mathrm{~s}^{-1}$ the width of our profiles is increased up to $\sim 400 \mathrm{~m} \AA$. A comparison between the spatially averaged spectrum with and without micro-turbulence is given in Fig. 2.

After adding the micro-turbulence the formal solution of the polarized radiative transfer equation was computed with Nicole using a Hermitian method, developed by Bellot Rubio et al. (1998). This two-step process from computing the Stokes profiles is necessary because MuLTi3D cannot compute polarized radiative transfer, and Nicole cannot compute 3D radiative transfer. Only by combining the two codes we obtain full Stokes profiles computed with $3 \mathrm{D}$ radiative transfer that serve as our synthetic observations for the inversion.

Our synthetic intensity profiles are near-identical to the results of Leenaarts et al. (2009), with small remaining differences due to small differences in the radiative transfer codes. In Fig. 3, monochromatic images at $\Delta \lambda=14.9,0.84,0.24,0.16$ and $0.00 \AA$ from the core of the line illustrate the vast formation range of the Ca II $\lambda 8542 \AA$ line, which covers the photosphere and part of the chromosphere. The polarization profiles are induced by Zeeman splitting. The Stokes $Q$ and $U$ profiles show amplitudes about $10^{-4}$ (relative to the continuum intensity), peaking around $10^{-3}$ at locations where the magnetic field is (relatively) strong and mostly horizontally oriented. The Stokes $V$ signal is one order of magnitude stronger than Stokes $Q$ and $U$, usually within the range $\left[10^{-3}, 10^{-2}\right]$, and shows extended areas with the same polarity.

The last column in Fig. 3 illustrates for each wavelength the height where the monochromatic optical depth equals one, $Z\left(\tau_{v}=1\right)$. In the wings of the line, the height of the $\tau_{v}=1$ surface is dominated by the temperature sensitivity of the opacity. This gives rise to granular "hills" with a higher $\tau_{v}=1$ surface than in the cooler intergranular lanes. Additionally, plasma evacuation in magnetic elements reduces the opacity, allowing to see deeper into the atmosphere (e.g. Keller et al. 2004). At $\Delta \lambda=0.837 \AA$ we sample the inverse granulation layer where the temperature contrast is lower and most of the variation in the height of the $\tau_{v}=1$ surface is caused by the lower plasma density where the magnetic field is strong. Closer to line center there is a strong variation in the $\tau_{v}=1$ surface caused by strong temperature and density variations and Doppler shifts of the line core into or out of the passband.

\section{Observability of Stokes signals}

This study is partially motivated by the instrumental requirements of future telescopes. In this context, Fabry-Pérot Interferometers (FPI) have become very popular because they allow a balanced trade-off between cadence, spatial resolution and spectral resolution. We analyze here the combined effects of limited spectral resolution and noise in our synthetic observations.

We assumed a Gaussian spectral filter transmission and additive photon noise produced by the finite photon flux. The instrumental point spread function (PSF) is characterized by its FWHM, and hereafter we refer to this parameter when instrumental spectral smearing is discussed. The additive noise is characterized in terms of its standard deviation $\sigma$ relative to the continuum intensity.

Our results are illustrated in Figs. 4 and 5 at $+205 \mathrm{~m} \AA$ from the line core, where the red peak of polarization is, on average, located.

In Fig. 4 we show the Stokes $Q$ signal in a subfield of the synthetic image for various combinations of spectral degradation and noise. The spectral degradation spans the range $[0,100] \mathrm{m} \AA$, in $25 \mathrm{~m} \AA$ steps, the noise levels are $\sigma=0,10^{-4}, 10^{-3.5}$ and $10^{-3}$. The noise value and spectral degradation are indicated inside each panel.

In the top row of Fig. 4 only spectral degradation is applied. Even though the amplitude of Stokes $Q$ is decreased by a factor 2 between $F W H M=0 \mathrm{~m} \AA$ and $F W H M=100 \mathrm{~m} \AA$, the main features are still visible at maximum spectral degradation.

The middle rows (panels $\mathrm{f}-\mathrm{o}$ ) correspond to $\sigma=10^{-4}$ and $\sigma=10^{-3.5}$. Stokes $Q$ features are still above the noise for all assumed values of the FWHM. This result is somewhat expected because our Stokes $Q$ profiles usually peak within $\left[10^{-4}, 10^{-3}\right]$, so noise only affects the very weak polarization signals.

The bottom row of Fig. 4 corresponds to $\sigma=10^{-3}$, where noise clearly dominates the Stokes $Q$ images. Some regions with strong polarization in $Q$ are discernible in panels $\mathrm{p}$ and $\mathrm{q}$ $(F W H M \leq 25 \mathrm{m \AA})$ but are barely visible when $F W H M=$ $50 \mathrm{~m} \AA$. Above this value, Stokes $Q$ vanishes below the noise because of spectral degradation.

Figure 5 shows Stokes $V$ images for the same subfield as Fig. 4, for the same values of spectral degradation. Only the case corresponding to $\sigma=10^{-3}$ is shown because the signal is well above the noise in all other cases.

Above, we have described and discussed several combinations of noise and spectral resolution. However, for a given telescope aperture, the signal-to-noise ratio can be derived as a function of the instrumental efficiency, pixel size and exposure time. To estimate the number of photons that can be detected at the core of the Ca II $\lambda 8542 \AA$ line, we computed the continuum intensity at $8542 \AA$ using Multi (see Carlsson 1986) and the FALC atmospheric model by Fontenla et al. (2006). The intensity ratio between the continuum and the core of the 


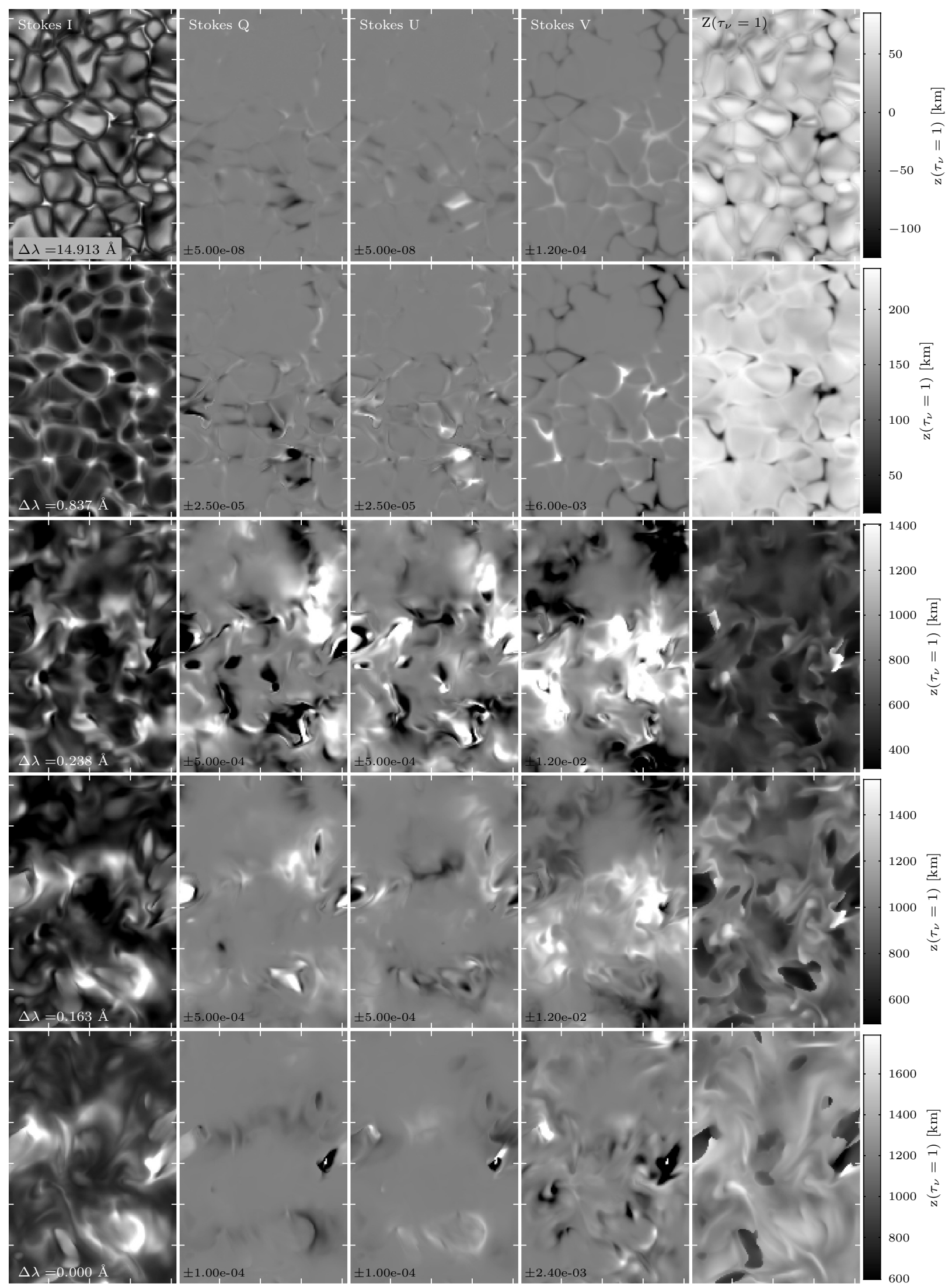

Fig. 3. Synthetic full-Stokes images computed from a $12.45 \times 8.3 \mathrm{Mm}$ patch of the model. From left to right, the first 4 columns show monochromatic Stokes $I, Q, U$ and $V$ images, respectively. The last column shows the height where the monochromatic optical depth equals one. From top to bottom, the wavelength decreases from the far red wing to the core of the line. The wavelength relative to line core is indicated on each of the Stokes $I$ panels. The Stokes $Q, U$ and $V$ panels have been scaled independently to enhance visibility of weak polarization features; the scale range is indicated within each panel in units of the continuum intensity. The tick-mark separation is $2 \mathrm{Mm}$. The panels have been transposed with respect to the panel orientation of Fig. 1. 
J. de la Cruz Rodríguez et al.: Non-LTE inversions from a 3D MHD chromospheric model

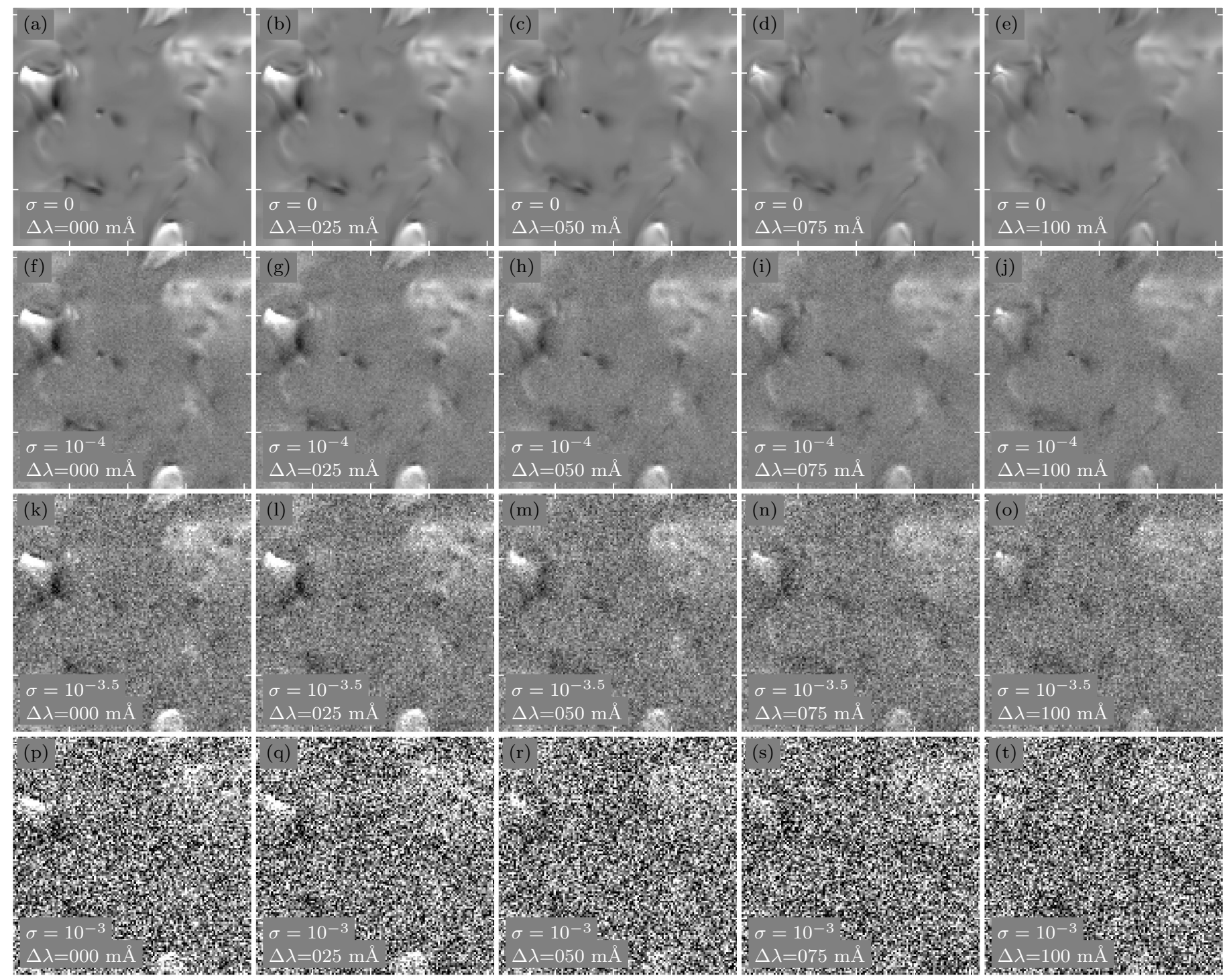

Fig. 4. Stokes $Q$ image spectrally degraded with Gaussian PSFs and random noise. From left to right, the spectral degradation increases as a function of the FWHM of the Gaussian, from $\Delta \lambda=0$ (no spectral smearing) to $\Delta \lambda=100 \mathrm{~m} \AA$. From top to bottom, the noise component increases, from $\sigma=0$ to $\sigma=10^{-3}$. The images are scaled to $\pm 0.15 \%$ relative to the continuum intensity.
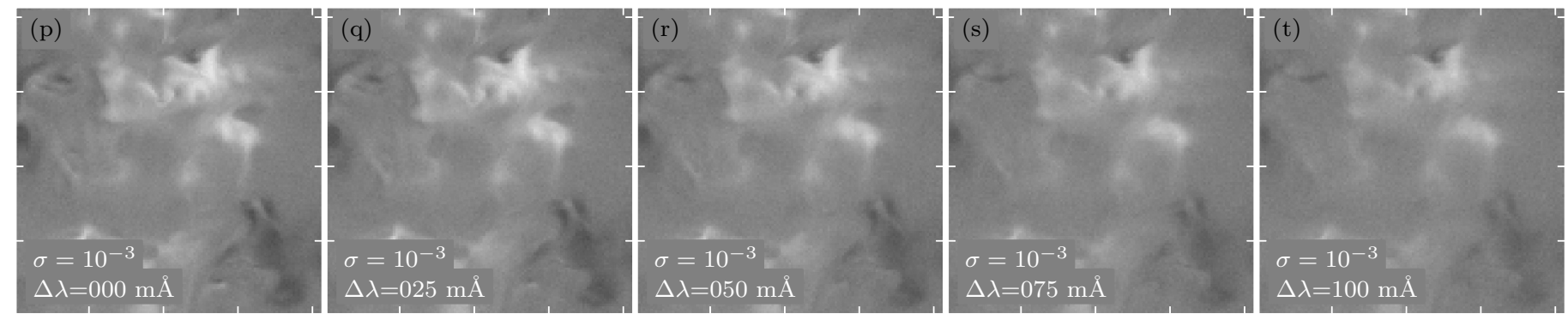

Fig. 5. Stokes $V$ image spectrally degraded with Gaussian PSFs. From left to right, the spectral degradation increases as a function of the FWHM of the Gaussian, from $\Delta \lambda=0$ (no spectral smearing) to $\Delta \lambda=100 \mathrm{~m} \AA$. A Gaussian random noise component of $\sigma=10^{-3}$ has been added. The images are scaled to $\pm 5 \%$ relative to the continuum intensity.

line $\left(I_{0}\right)$ is derived from the Fourier transform spectrometer at the McMath-Pierce Telescope (hereafter called the FTS atlas) of Brault \& Neckel (1987), resulting in $I_{0}=I_{\mathrm{lc}} / I_{\text {cont }}=0.18$. For a telescope aperture of $1.5 \mathrm{~m}$, a spectral resolution of $R=200000$ (42 $\mathrm{m} \AA$ at 18542 ), a pixel size of $0.1^{\prime \prime}$ and an overall system efficiency of 0.1 (telecope-instrument-detector), one can detect $n_{0}=2.66 \times 10^{6}$ photons per second. This corresponds to a photon noise level of $6.1 \times 10^{-4}$.

Figure 6 illustrates similar results for several combinations of exposure times ( $\infty \mathrm{s}, 16 \mathrm{~s}, 4 \mathrm{~s}$ and $1 \mathrm{~s})$ and spectral degradation (0 $\mathrm{m} \AA, 25 \mathrm{~m} \AA, 50 \mathrm{~m} \AA$ and $100 \mathrm{~m} \AA$ ). One can see the difficulties that arise from having a too high spectral resolution: as the 

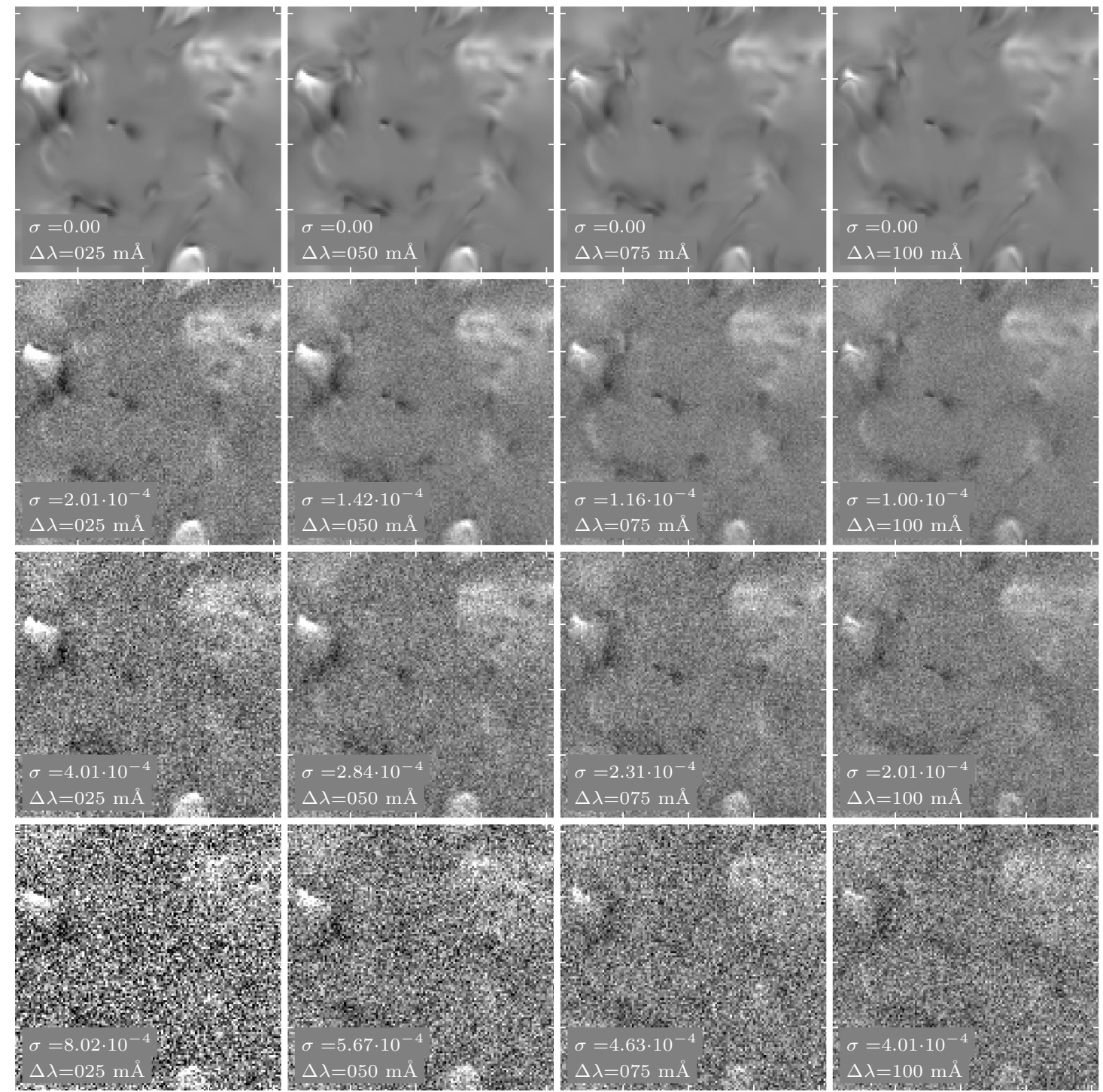

Fig. 6. Stokes $Q$ image spectrally degraded with Gaussian PSFs and random noise. From left to right, the spectral degradation increases as a function of the FWHM of the Gaussian, from $\Delta \lambda=25 \mathrm{~m} \AA$ to $\Delta \lambda=100 \mathrm{~m} \AA$. From top to bottom, the integration time is $\infty, 16 \mathrm{~s}, 4 \mathrm{~s}$ and $1 \mathrm{~s}$, respectively. The images are scaled to $\pm 0.15 \%$ relative to the continuum intensity. For a fixed exposure time the noise in each image increases with decreasing width of the instrumental profile.

Table 1. Photon noise levels as a function of the exposure time and the spectral resolution.

\begin{tabular}{l|cccc}
\hline \hline & $25 \mathrm{~m} \AA$ & $50 \mathrm{~m} \AA$ & $75 \mathrm{~m} \AA$ & $100 \mathrm{~m} \AA$ \\
\hline $16 \mathrm{~s}$ & $2.00 \times 10^{-4}$ & $1.42 \times 10^{-4}$ & $1.16 \times 10^{-4}$ & $1.00 \times 10^{-4}$ \\
$4 \mathrm{~s}$ & $4.00 \times 10^{-4}$ & $2.83 \times 10^{-4}$ & $2.31 \times 10^{-4}$ & $2.00 \times 10^{-4}$ \\
$1 \mathrm{~s}$ & $8.02 \times 10^{-4}$ & $5.67 \times 10^{-4}$ & $4.63 \times 10^{-4}$ & $4.00 \times 10^{-4}$ \\
\hline
\end{tabular}

instrumental transmission profile becomes narrower, polarization features become more prominent but the noise is also significantly higher. By increasing the exposure time, noise is decreased, but as we explain below, image degradation can appear from the rapid chromospheric motions. Additionally, higher spectral resolution increases the number of wavelength points that are needed to sample the line profile. For clarity, Table 1 summarizes the numbers used in Fig. 6.

The FPI instruments that are currently operating in solar telescopes can typically achieve a spectral resolution of 45-100 m $\AA$ in the near-infrared, with noise levels of about $10^{-3}$. Those cases correspond to panels r, s and t in Figs. 4 and 5. Our results suggest that Stokes $V$ detections are possible with current instrumentation. However, the detection of linear polarization in the quiet Sun requires sensitivities of at least $10^{-3.5}$.

Exposure time in solar observations is limited by the evolution time of the Sun. Longer exposures than the evolution time scale can produce smearing of the data. Particularly, image reconstruction assumes that the Sun is static for a given set of short exposure images. The vigorously dynamic chromosphere shows features moving horizontally within one second (van Noort \& Rouppe van der Voort 2006). Additionally, FPI instruments can only observe one wavelength at the time, a limiting factor for the wavelength coverage given that all acquisitions within one line scan should be as simultaneous as possible. Therefore observing the chromosphere involves a difficult trade-off between wavelength coverage and exposure time.

There are ways to ameliorate the sampling. The broad Ca II infrared lines have a wide formation range: the fast-moving features observed in the cores contrast with the slow evolution 
J. de la Cruz Rodríguez et al.: Non-LTE inversions from a 3D MHD chromospheric model

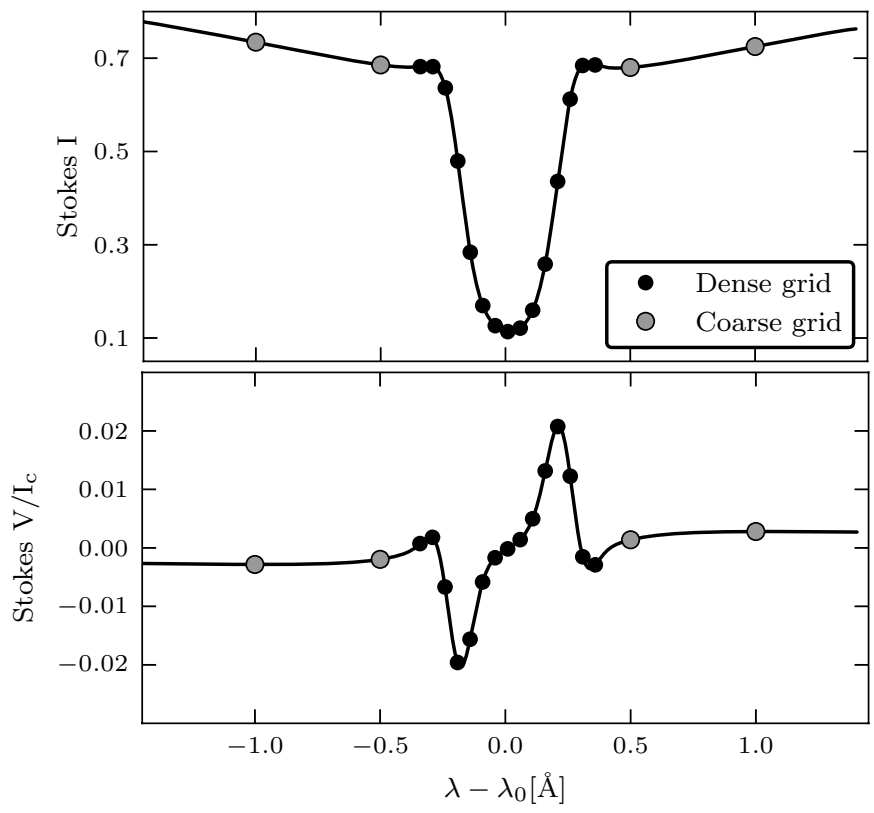

Fig. 7. Stokes $I$ and $V$ profiles from one of the columns from the 3D model. The instrumental spectral FWHM is $100 \mathrm{~m} \AA$. The gray circles indicate a coarse grid of points located outside of the chromospheric range of the line. The black circles sample the core of the line every $50 \mathrm{m \AA}$.

of photospheric granulation present in the wings of the lines. Additionally, the amplitude of Stokes $Q, U$ and $V$ rapidly decreases toward the wings of the line.

A strategy to optimize the polarization measurements and achieve proper time sampling would be to place a fine grid of points in the chromospheric core and a coarser grid in the wings, where slower variations are expected. Because the photosphere evolves on a longer time scale than the chromosphere, exposures in the wings do not have to be recorded as frequently as in the core. Figure 7 illustrates this scheme, with a fine grid of points (black circles) distributed in the core every $50 \mathrm{~m} \AA$ and a coarser grid in the wings (gray circles) placed every $500 \mathrm{~m} \AA$.

\section{Inversions}

We used the code Nicole for the inversions presented in this section. It solves the NLTE problem assuming plane-parallel geometry, isotropic scattering and complete frequency redistribution, using the strategy described by Socas-Navarro \& Trujillo Bueno (1997). All Zeeman sublevels originating from a given atomic level are assumed to be equally populated, discarding any quantum interference between them, as proposed by Trujillo Bueno \& Landi Degl'Innocenti (1996).

The velocity-free approximation is used for our calculations here, which assumes a static atmosphere when the atomicpopulations are computed. Under these conditions, only half of the profile needs to be computed and fewer quadrature angles are needed. Once the NLTE populations are calculated, the velocity stratification is taken into account to produce the final emerging profiles.

The inversions are initialized with a guess model that contains temperature, electron pressure, line-of-sight (l.o.s.) velocity, micro-turbulence and the three components of the magnetic field vector. The inversion engine of NICOLE is based on a Levenberg-Marquardt algorithm that computes the corrections

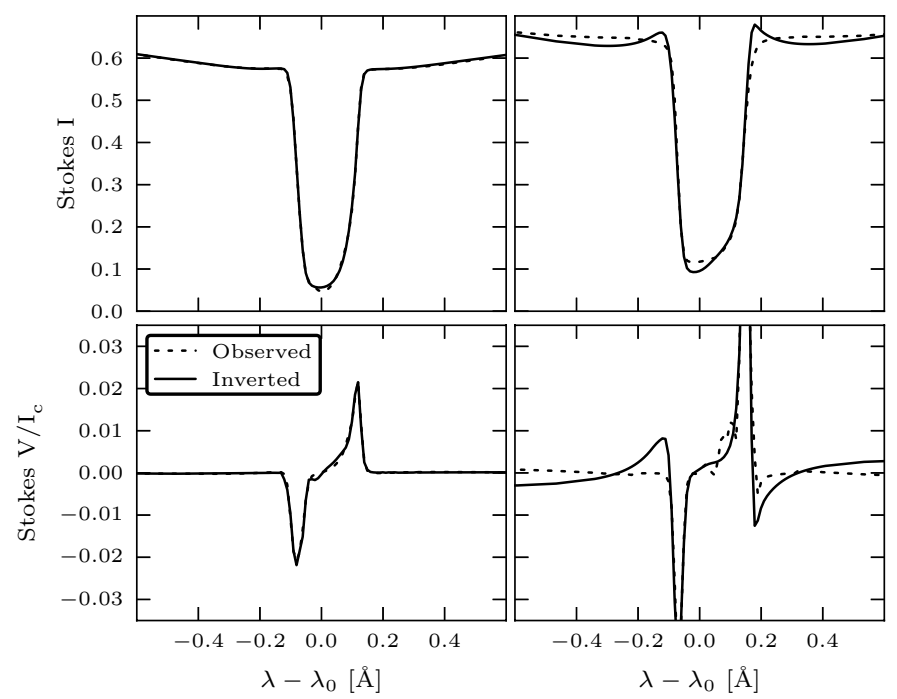

Fig. 8. Illustrative examples of the fits (solid line) to the simulated observations (dashed line) at two different locations in the LTE inversion of Fig. 14. Left panels: a good fit, at pixel coordinates $(25,23)$ in Fig. 14. Right panels: a comparatively poorer fit at pixel coordinates $(39,34)$. Upper row: Stokes $I$ (normalized to the average continuum intensity). Lower row: Stokes $V$.

to a guess model in order to minimize the differences between the observed and the synthetic profiles. To maintain physical consistency between temperature, electron pressure and gas pressure, the model is set to hydrostatic equilibrium after each correction. For a given temperature, the gas pressure and the electron pressure are obtained using an equation of state and the hydrostatic equilibrium approximation.

The corrections to the guess model were applied at node points that are equidistantly distributed over the depth scale of the model. The first inversion was initialized with the VAL-C model. After the first inversion another five inversions were performed starting from the VAL-C model where the atmospheric quantities have been given a random offset. This minimized the odds of the algorithm settling into a local minimum of the $\chi^{2}$ hypersurface.

Figure 8 shows the difference in the fit between a pixel that converged to the correct solution and another one for which the algorithm settled in a secondary minimum of the $\chi^{2}$ hypersurface. The noise created in the resulting image by imperfect fits (we will refer to this as inversion noise to avoid confusion with the usual photon noise found in the observations) can be minimized by implementing more sophisticated schemes with multiple initializations to ensure that one reaches the absolute minimum. Genetic algorithms (Charbonneau 1995) provide a suitable approach to the problem and have been successfully implemented in Stokes inversion codes (e.g., Lagg et al. 2004). However, such an algorithm dramatically increases the already very large amount of computational work required for the solution of the NLTE inversion problem. For this reason we have refrained from implementing anything beyond the already mentioned five attempts with randomized initializations.

The spectral resolution of the instrument, the wavelength coverage and sampling and the signal-to-noise ratio set constraints on the amount of information that can be retrieved by an inversion. For a given set of these quantities there is a maximum number of nodes after which adding more nodes does not improve the inversion of the data. Adding more nodes can lead instead to artifacts. 
Table 2. Summary of node points used during each cycle of the inversion.

\begin{tabular}{l|cc}
\hline \hline Physical parameter & Nodes in cycle 1 & Nodes in cycle 2 \\
\hline Temperature & 6 & 10 \\
l.o.s velocity & 2 & 5 \\
$B_{z}$ & 1 & 4 \\
$B_{x}$ & 0 & 1 \\
$B_{y}$ & 0 & 1 \\
\hline
\end{tabular}

To improve convergence, the inversions were computed in two cycles (see Ruiz Cobo \& del Toro Iniesta 1992; Pietarila et al. 2007; Socas-Navarro 2011). In the first cycle, a limited set of nodes was used to obtain a rough solution that was then refined during the second cycle with more nodes. A summary of the number of nodes used on each cycle is shown in Table 2.

The four Stokes parameters are weighted differently in the inversion. The following ratio was used in the present work: $W(I, Q, U, V)=[1,20,20,8]$.

The inversions were computed with critically sampled profiles. For spectral resolutions of $50 \mathrm{~m} \AA$ and $100 \mathrm{~m} \AA$, the spectral sampling at the chromospheric core are $25 \mathrm{~m} \AA$ and $50 \mathrm{~m} \AA$, respectively. The photospheric line wings are covered with a coarse grid of points separated by $1 \AA$ in all cases.

\subsection{Non-LTE inversions}

We performed NLTE inversions of our simulated Stokes profiles, which were first convolved with a Gaussian function of 50 FWHM, without noise.

The results of these inversions are compared with the original atmosphere in Fig. 9. Each quantity that is shown is the average over height between $\log _{10}\left(\tau_{500}\right)=-4.5$ and the depth where the transition region starts at each pixel, on average $\log _{10}\left(\tau_{500}\right)=-5.52$. The inversion code usually fails to reproduce the steep temperature gradient of the transition region, therefore it has been excluded from the depth average. In the $3 \mathrm{D}$ snapshot, the $\log _{10}\left(\tau_{500}\right)=-4.5$ iso-surface corresponds on average to $z=737 \pm 83 \mathrm{~km}$; the $\log _{10}\left(\tau_{500}\right)=-5.52$ iso-surface is located on average at $z=1340 \pm 365 \mathrm{~km}$. We stress that each column has its own run of $\tau_{5000}$ with height in the atmosphere. The derived quantities can therefore not be directly interpreted as the average over height in a horizontal slab.

The first row in Fig. 9 compares temperatures. The inversion reproduces most of the high-temperature structure, but fails to reproduce the low temperatures. This failure is at least partly explained by the effect of $3 \mathrm{D}$ radiation, which is not taken into account in the inversion, because this assumes each pixel is a 1D plane-parallel atmosphere. Despite the failure to reproduce low temperatures, the average relative error in the inferred temperature (measured as the difference between model and inversion divided by the model) is only $6.7 \%$. The Pearson correlation coefficient for the temperature is $r=0.51$. If we exclude cold areas $(T<3500 \mathrm{~K})$, the correlation factor becomes $r=0.67$.

The second row of Fig. 9 shows the line-of-sight velocity. The inversion is able to recover almost the entire structure originally present in the simulation, with a Pearson correlation coefficient of $r=0.80$. The differences between the model and the inversion are mainly caused by inversion noise. Although in this paper we explore the inversion of one spectral line, it is still possible to retrieve information about gradients (mostly on the lineof-sight velocity and the magnetic field) because these gradients are needed to reproduce the line asymmetries. The extreme case of a shock propagating through the atmosphere is discussed below in more detail.

The third row shows the vertical component of the magnetic field. The inversion reproduces the values in the atmosphere very well, with a Pearson correlation coefficient of $r=0.97$. The fourth and fifth row finally show the horizontal components of the magnetic field. The quality of the reconstruction is lower than for $B_{z}$, but still very good, with correlation coefficients of $r=0.90$ for $\left|B_{x}\right|$ and 0.78 for $\left|B_{y}\right|$. We note the presence of some artifacts in the inverted $\left|B_{x}\right|$ and $\left|B_{y}\right|$ maps, such as in $\left|B_{x}(x=6.5, y=4.5)\right|$, where the inverted field is stronger than in the $3 \mathrm{D}$ snapshot. These artifacts appear because the inversion is computed here with one node in $\left|B_{x}\right|$ and one node in $\left|B_{y}\right|$. The presence of strong photospheric fields can pollute the inverted values. Unfortunately, increasing the number of nodes also increases the inversion noise.

Figure 10 shows probability density plots comparing the inverted quantities with the original data. All inverted quantities show a tight correlation with the original data, except for the temperature.

The failure to reproduce low temperature areas is further illustrated in Fig. 11. The top panel compares the emerging intensity profile for a pixel with a cold chromosphere (shown in the lower panel) computed in $3 \mathrm{D}$ and in $1 \mathrm{D}$. In $1 \mathrm{D}$, the linecore intensity is low, as a consequence of the low temperature. In 3D, however, the cold gas is illuminated from the sides, which increases the source function and hence the emerging intensity. The lower panel shows the temperature in the model atmosphere, and the temperature from the inversion. The inversion shows a much higher temperature (and hence source function) to reproduce the relatively high 3D line-core intensity.

The presence of shocks in the atmosphere poses a particular problem for the inversion. Owing to the limited number of nodes, the inversion will often not be able to recover the true atmospheric structure. Instead it will try to find a much smoother structure that nevertheless approximates the emerging intensity.

We illustrate this with an example from the $3 \mathrm{D}$ simulation in Fig. 12: the shock is located between $\log _{10}\left(\tau_{500}\right)=-5$ and $\log _{10}\left(\tau_{500}\right)=-6$, as indicated by the increasing temperature and a spike in the vertical velocity. Together these lead to an asymmetric line core. The profile minimum is blueshifted with respect to the rest line-center frequency and the profile shows an inflection point at $\Delta \lambda=0.16 \AA$.

The profile-minimum intensity is well reproduced by the inversion, as is the profile-minimum Doppler shift, but the inflection point is not recovered. That the profile-minimum intensity is recovered means that the inferred temperature follows the true temperature in the shock region. The atmospheric velocity is not accurately recovered: the limited number of nodes forces a smooth inferred velocity field, which yields the same profileminimum Doppler shift as the forward calculation, but has a lower maximum velocity. The inferred velocity has a smooth transition from 2 to $0 \mathrm{~km} \mathrm{~s}^{-1}$ between $\log _{10}\left(\tau_{500}\right)=-5$ and $\log _{10}\left(\tau_{500}\right)=-4$, whereas the atmosphere has a much steeper velocity gradient. The lack of this steep gradient causes the lack of the inflection point in the inverted profile.

Figure 13 shows the effect of spectral resolution and photon noise on the quality of the inversions. Because of the large amount of computational work, we restricted the inversions to an area of $4 \times 2.5 \mathrm{Mm}^{2}$ with its lower left corner at $(x, y)=$ $(6,3) \mathrm{Mm}$ in Fig. 9. We have introduced Gaussian noise of $\sigma=10^{-4}, 10^{-3.5}$ and $10^{-3}$. Comparison of the left-hand and right-hand mosaics shows that the quality of the inversion is more affected by noise levels than by the spectral degradation 
J. de la Cruz Rodríguez et al.: Non-LTE inversions from a 3D MHD chromospheric model

Inversion
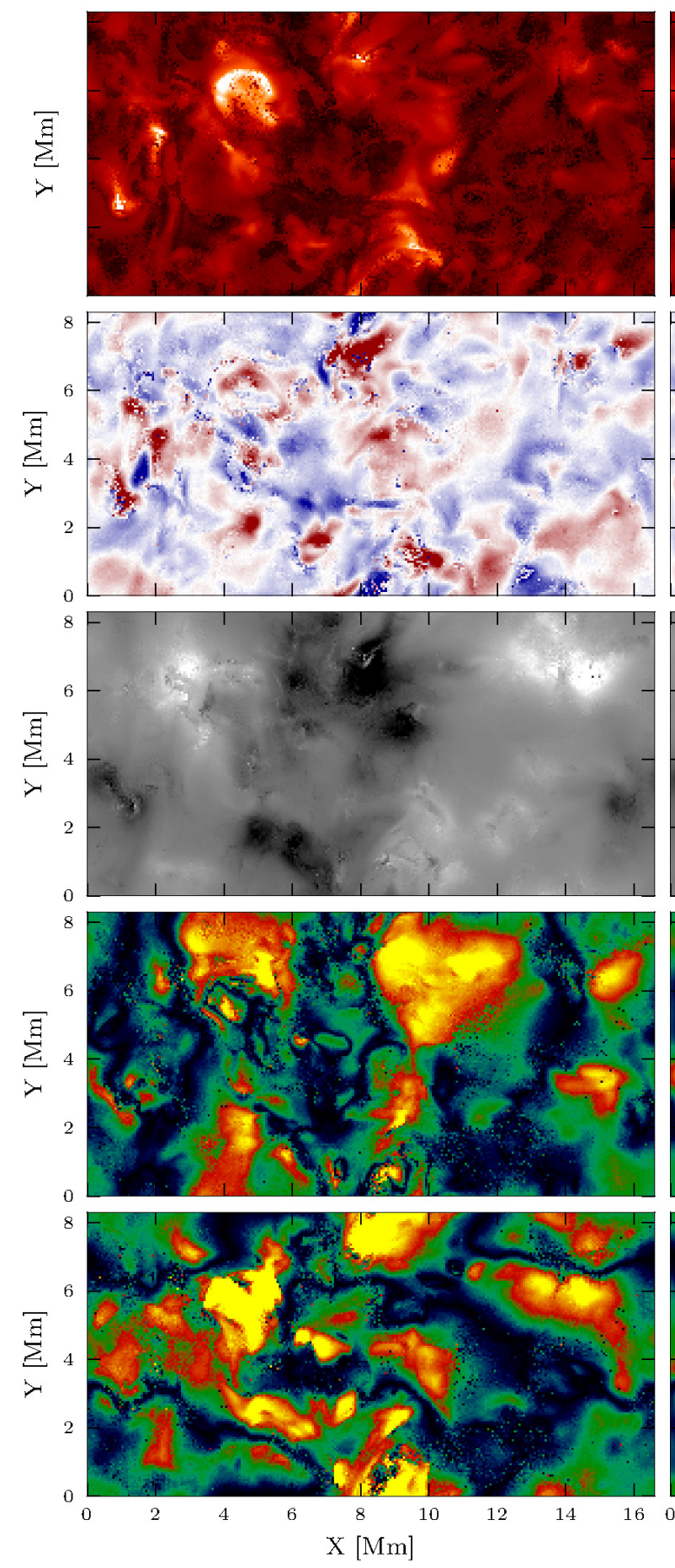

3D snapshot
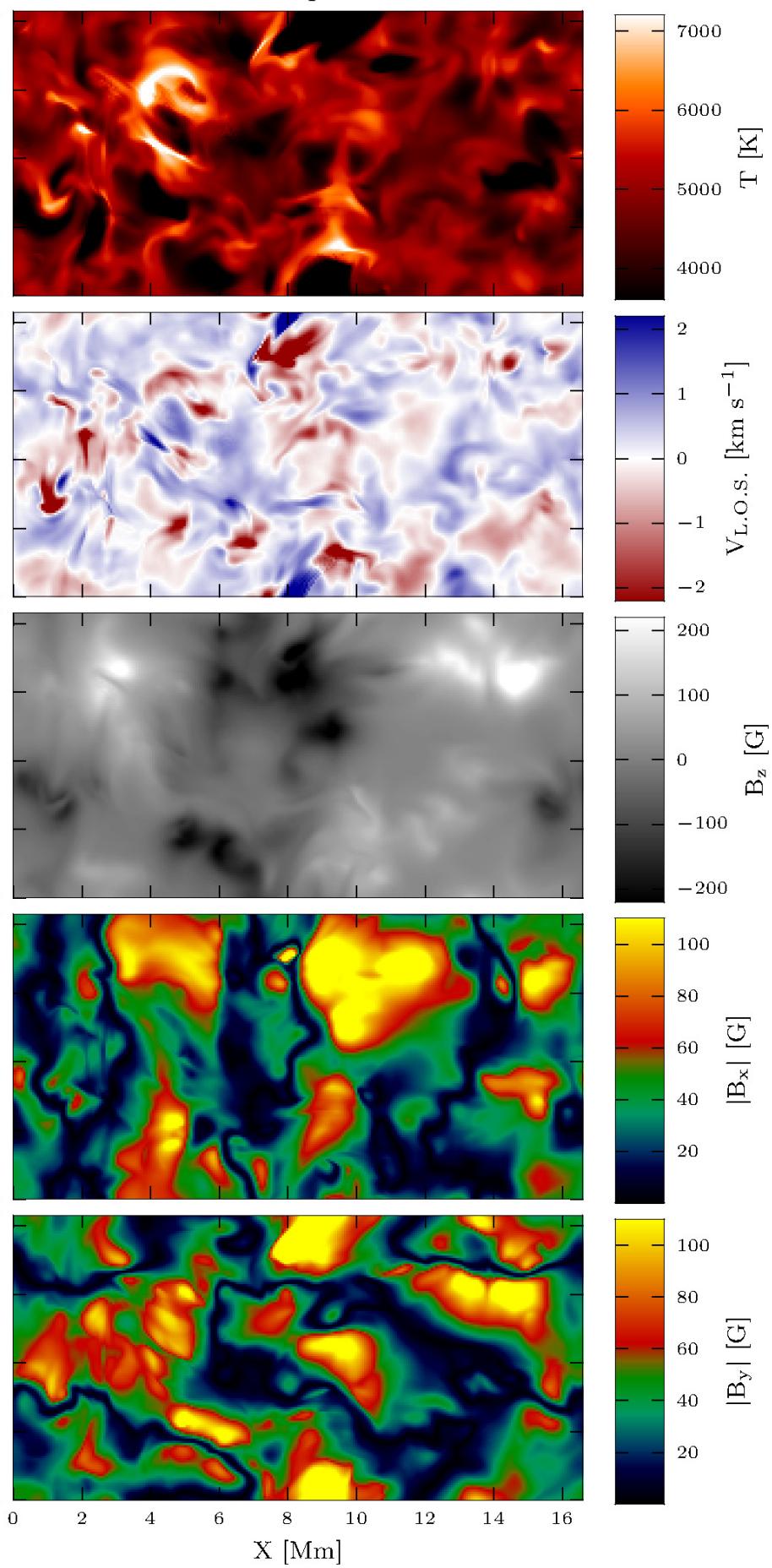

Fig. 9. Full field-of-view comparison between physical quantities in the chromosphere as determined from the NLTE inversion (left) and the original 3D snapshot (right). From top to bottom: temperature, line-of-sight velocity, $B_{z},\left|B_{x}\right|$ and $\left|B_{y}\right|$. The line profiles were degraded with an instrumental profile of $F W H M=50 \mathrm{~m} \AA$ before carrying out the inversion. In the velocity panel, upflows have positive sign.
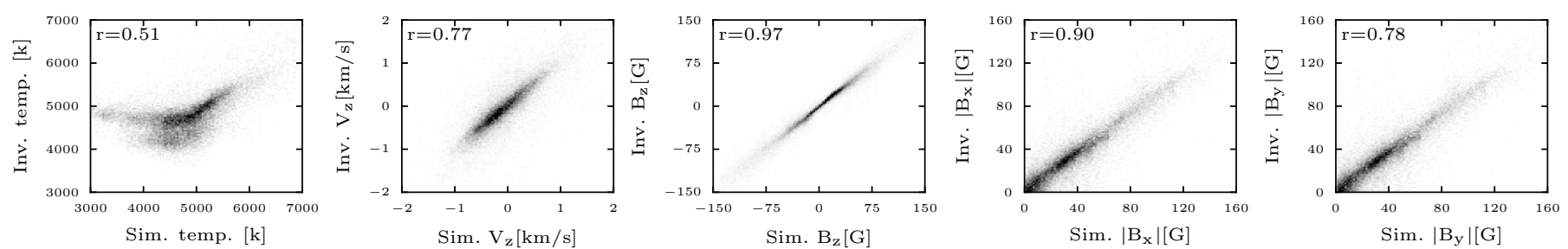

Fig. 10. Probability density plots of the inverted quantities vs. the $3 \mathrm{D}$ simulation quantities. From left to right: temperature, $V_{z}, B_{z},\left|B_{x}\right|$ and $\left|B_{y}\right|$. The Pearson correlation coefficient $(r)$ is indicated in the top-left corner of each panel. 

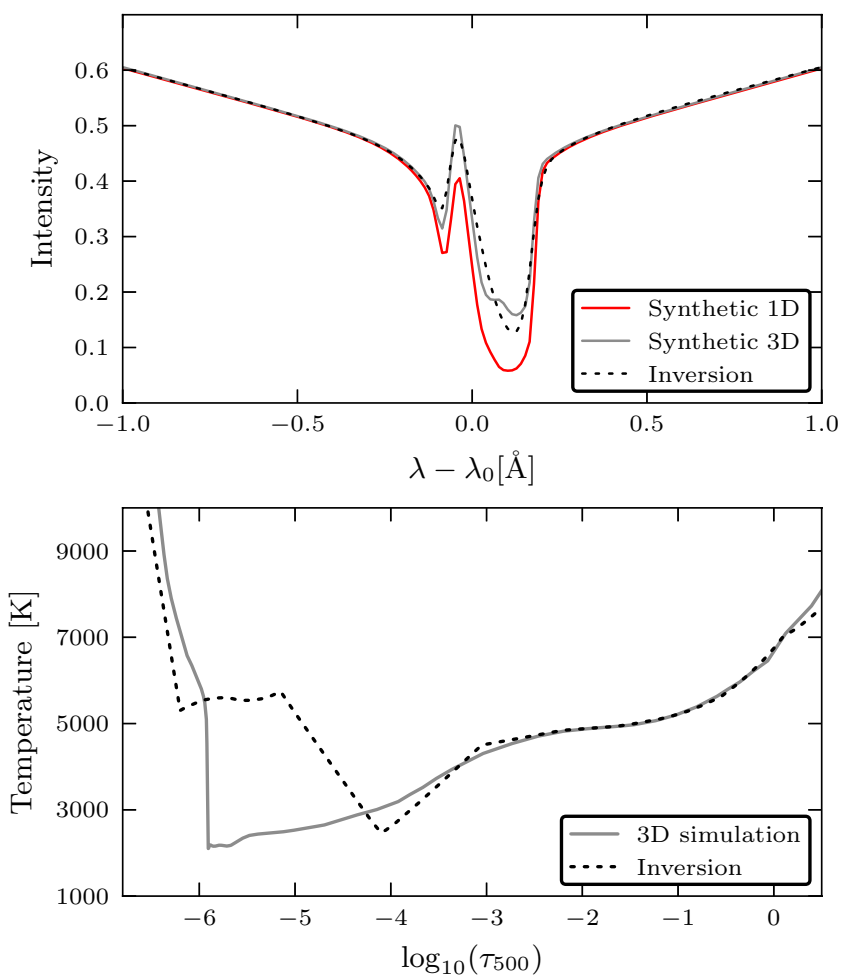

Fig. 11. Upper panel: vertically emerging intensity profile for a pixel with a low chromospheric temperature computed with MuLri3D using $3 \mathrm{D}$ radiative transfer (solid-gray), the profile for the same pixel, but assuming 1D plane-parallel geometry (solid-red) and the best fit from an inversion (dashed). Lower panel: the temperature structure for the same pixel (solid-gray) and the temperature structure inferred from the inversion (dashed).

considered in our inversions. For a given noise level, the results obtained for a spectral resolution of $50 \mathrm{~m} \AA$ are slightly better than at $100 \mathrm{~m} \AA$.

The longitudinal magnetic field $\left(B_{z}\right)$ is the most accurately retrieved parameter from the inversions. The Stokes $V$ profiles usually peak well above the $10^{-3}$ level. The reconstructed longitudinal field becomes increasingly noisy with increasing photon noise, but the overall structure remains the same.

The maximum synthetic Stokes $Q$ and $U$ signal is typically only $5 \times 10^{-4}$. Reconstruction of the horizontal components of the magnetic field is therefore much more sensitive to the noise level. At $10^{-4}$ noise, the inversion is able to recover most of the structure present in the atmosphere, more successfully when the $F W H M=50 \mathrm{~m} \AA$. When noise is increased to $\sigma=10^{-3.5}$, most of the details are lost, but areas with horizontal field larger than $\sim 100 \mathrm{G}$ are still discernible. Finally, when the noise is of the order of $10^{-3}$, the inversion is unable to recover the transverse magnetic field components.

\subsection{Velocity and magnetic field in LTE}

The intensity absorption profile of a given spectral line is given by the expression (see, e.g. Landi Degl'Innocenti 1992)

$$
\begin{aligned}
\kappa_{I}\left(\lambda, T, \boldsymbol{B}, v_{\mathrm{los}}\right)= & \kappa_{\mathrm{C}}(T)+\frac{1}{2} \eta_{0}\left(T, n_{\mathrm{l}}, n_{\mathrm{u}}\right)\left[\phi_{p}\left(\lambda, \boldsymbol{B}, v_{\mathrm{los}}\right) \sin 2 \theta\right. \\
& \left.+\frac{\phi_{b}\left(\lambda, \boldsymbol{B}, v_{\mathrm{los}}\right)+\phi_{r}\left(\lambda, \boldsymbol{B}, v_{\mathrm{los}}\right)}{2}(1+\cos 2 \theta)\right]
\end{aligned}
$$
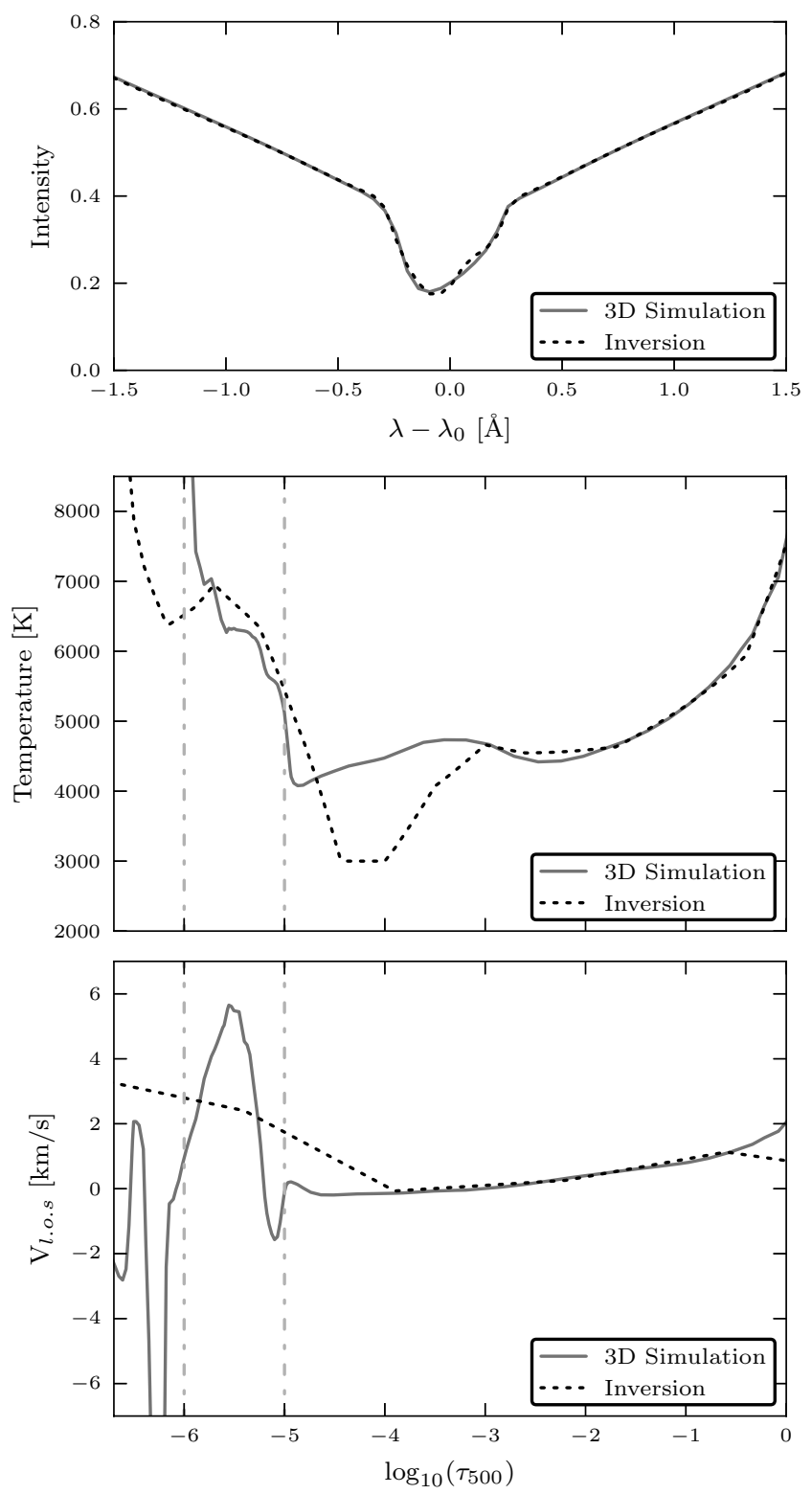

Fig. 12. Upper panel: intensity profile calculated from a column of the model where a shock is present (solid line). The dashed line corresponds to the inversion result. Middle panel: temperature stratification in the original model (solid) and the inversion result (dashed). Bottom: line-of-sight velocity stratification in the original model (solid) and the inversion result (dashed). The shock is located between the two gray dash-dotted lines.

where $\kappa_{\mathrm{C}}$ is the continuum absorption coefficient, $\eta_{0}$ is the line strength, $\phi_{p}, \phi_{b}$ and $\phi_{r}$ are the $\pi$, blue and red profile components, respectively. Equation (4) explicitly shows the dependencies on the temperature, $T$ (and other thermodynamical parameters such as density or pressure), magnetic field vector $\boldsymbol{B}$ and the line-of-sight velocity $v_{\mathrm{los}}$. For the continuum absorption coefficient $\kappa_{\mathrm{C}}$ we have neglected the wavelength dependence because the continuum opacity varies with wavelength much more slowly than the line opacity. The atomic level populations of the lower and upper levels of the transition considered, $n_{1}$ and $n_{\mathrm{u}}$, obviously depend on $T$ as well. Even in conditions far from LTE, the atomic populations are only weakly dependent on $\boldsymbol{B}$ and $v_{\text {los }}$, unless the velocity gradients are very steep.

In Eq. (4) there is a convenient separation of the atmospheric parameters (the unknowns of our inversions). The dependence 


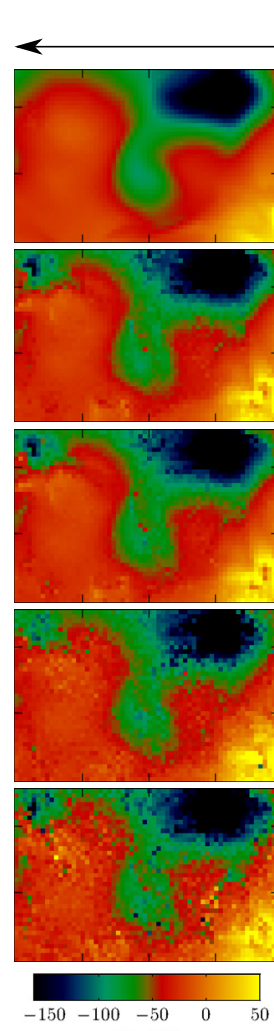

$\mathrm{B}_{\mathrm{z}}[\mathrm{G}]$
$\mathrm{FWHM}=50 \mathrm{m \AA}$
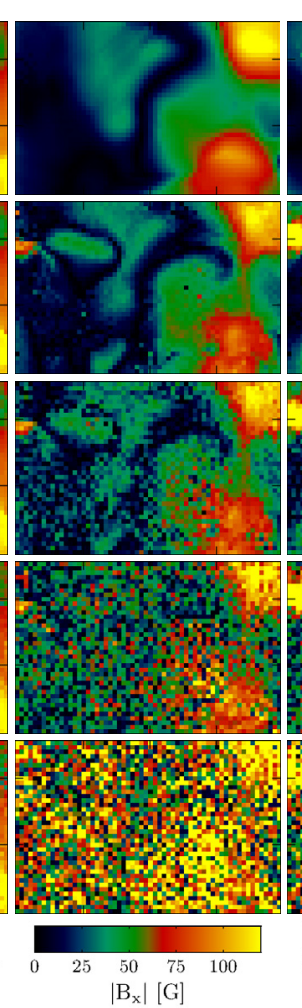
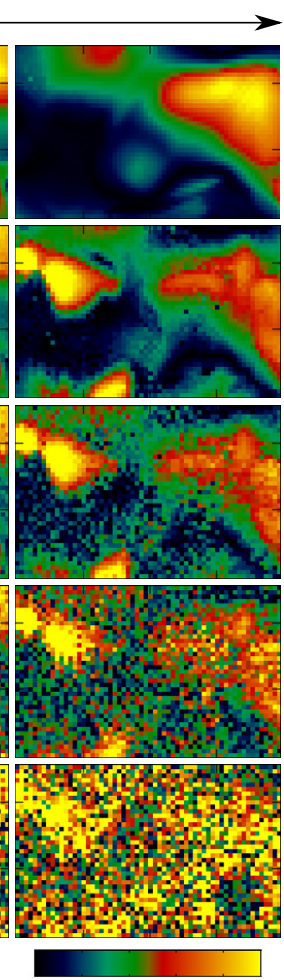

$\begin{array}{lllll}25 & 50 & 75 & 100\end{array}$

$\mathrm{B}_{\mathrm{y}} \mid[\mathrm{G}]$

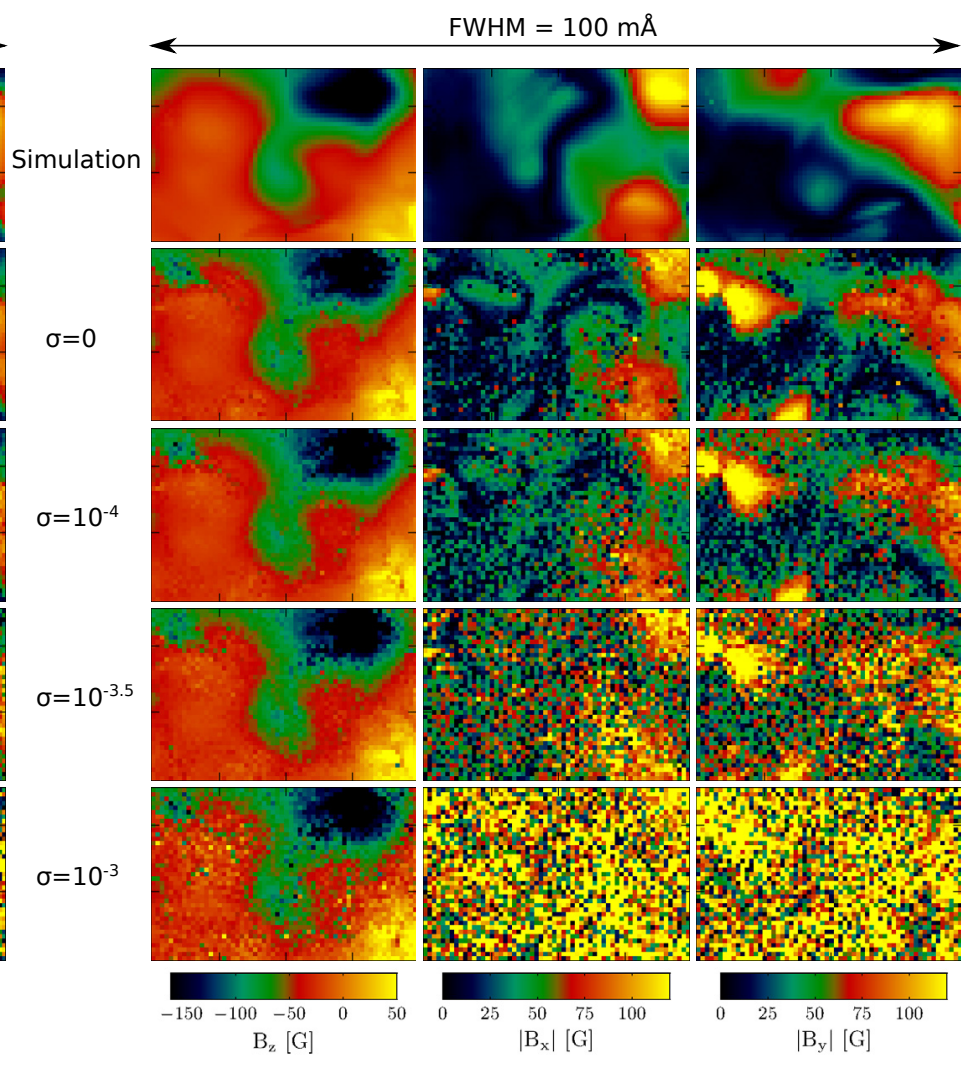

Fig. 13. Illustration of the effect of spectral resolution and photon noise on the quality of the inversions. The left-hand mosaic shows the results of the inversion after spectral degradation of the line profiles with a Gaussian instrumental profile with $F W H M=50 \mathrm{~m} \AA$, the right-hand panel after smearing with a profile with a FWHM of $100 \mathrm{~m} \AA$. Within each mosaic, from left to right, the panels show $B_{z}(x, y),\left|B_{x}(x, y)\right|,\left|B_{y}(x, y)\right|$. Rows, from top to bottom, show the magnetic field from the 3D snapshot, followed by the inversion results assuming random photon noise of $\sigma=0, \sigma=10^{-4}$, $\sigma=10^{-3.5}$ and $\sigma=10^{-3}$.

on the thermodynamical parameters $T$ is contained exclusively in $\kappa_{\mathrm{C}}$ and $\eta_{0}$, whereas the dependencies on the magnetic field $\boldsymbol{B}$ and the velocity $v_{\text {los }}$ are in the term enclosed in square brackets. We can then see that the wavelength dependence of the profile (which is contained in the terms within the brackets) depends only on the values of $\boldsymbol{B}$ and $v_{\text {los. }}$. The same happens with the equivalent absorption profiles for the rest of the Stokes parameters $\kappa_{Q}, \kappa_{U}, \kappa_{V}$, as well as the emission and the anomalous dispersion profiles. They can all be separated into a term containing the atomic populations and the thermodynamic properties and another term containing the wavelength profiles with the dependence on $\boldsymbol{B}$ and $v_{\text {los }}$.

This separation of variables is fortunate because if we are not interested in the temperature but only in the magnetic field and velocity, it is possible to devise a much simpler inversion for these quantities. This simpler inversion would not require the solution of an NLTE problem, and the computational demands are much lower.

Let us consider now one such simplistic procedure, namely one that fits the observed profiles computing the atomic populations in LTE. With the inversion we obtain a run of thermodynamic parameters $T^{*}$ which is, in general, different from the real $T$ in the solar atmosphere. From that $T^{*}$ we compute LTE populations $n_{1}^{*}$ and $n_{\mathrm{u}}^{*}$.

When the observations are fitted by the procedure, the source function and the opacity are adequately reproduced. Therefore, the populations obtained are approximately correct $\left(n_{1}^{*} \simeq n_{1}\right.$ and $n_{\mathrm{u}}^{*} \simeq n_{\mathrm{u}}$ ), even though the temperatures needed to yield those values are drastically different in LTE and NLTE. The wavelength dependence of the observed spectral line depends entirely on $\boldsymbol{B}$ and $v_{\text {los }}$, whose effects on the profile are the same regardless of whether the populations are computed in LTE or NLTE. In summary, with our simplistic LTE fitting procedure we would recover $\boldsymbol{B}$ and $v_{\text {los }}$ with the same degree of accuracy as with an NLTE inversion, and only $T$ would be wrong.

With the same reasoning, we argue that questioning the validity of the assumptions that we have made on the radiative transfer (such as hydrostatic equilibrium, 1.5D atmosphere or the assumption of statistical equilibrium) would only be relevant in determining $T$ but not $\boldsymbol{B}$ or $v_{\text {los }}$.

This approach is actually similar to what is often performed in Milne-Eddington inversions for the photosphere, in which only $\boldsymbol{B}$ and $v_{\text {los }}$ are interpreted as physical parameters, even though several other parameters are involved in the fit.

Figure 14 depicts the result of this LTE inversion of the synthetic Stokes profiles to determine $\boldsymbol{B}$ and $v_{\mathrm{los}}$ in the same region of the simulation as for Fig. 13. The inversions in Fig. 14 correspond to $\sigma=10^{-4}$ and a spectral resolution of $50 \mathrm{~m} \AA$. Comparison of the inferred $B_{z}$ and $v_{\operatorname{los}}$ shows that the inversion performs reasonably well in reproducing these quantities. The noise comes primarily from inversions that failed to converge. The Pearson correlation coefficients are $r=0.93$ for $B_{z}$ and $r=0.84$ for $v_{\text {los }}$.

For $B_{x}$ and $B_{y}$ the results appear more noisy than for $B_{z}$, but the inversion more or less correctly reproduces the patches of strong field on the right side of the panel. The inversion erroneously infers patches of horizontal field where there are none in the atmosphere, such as at $(x, y)=(12,28)$. The Pearson 

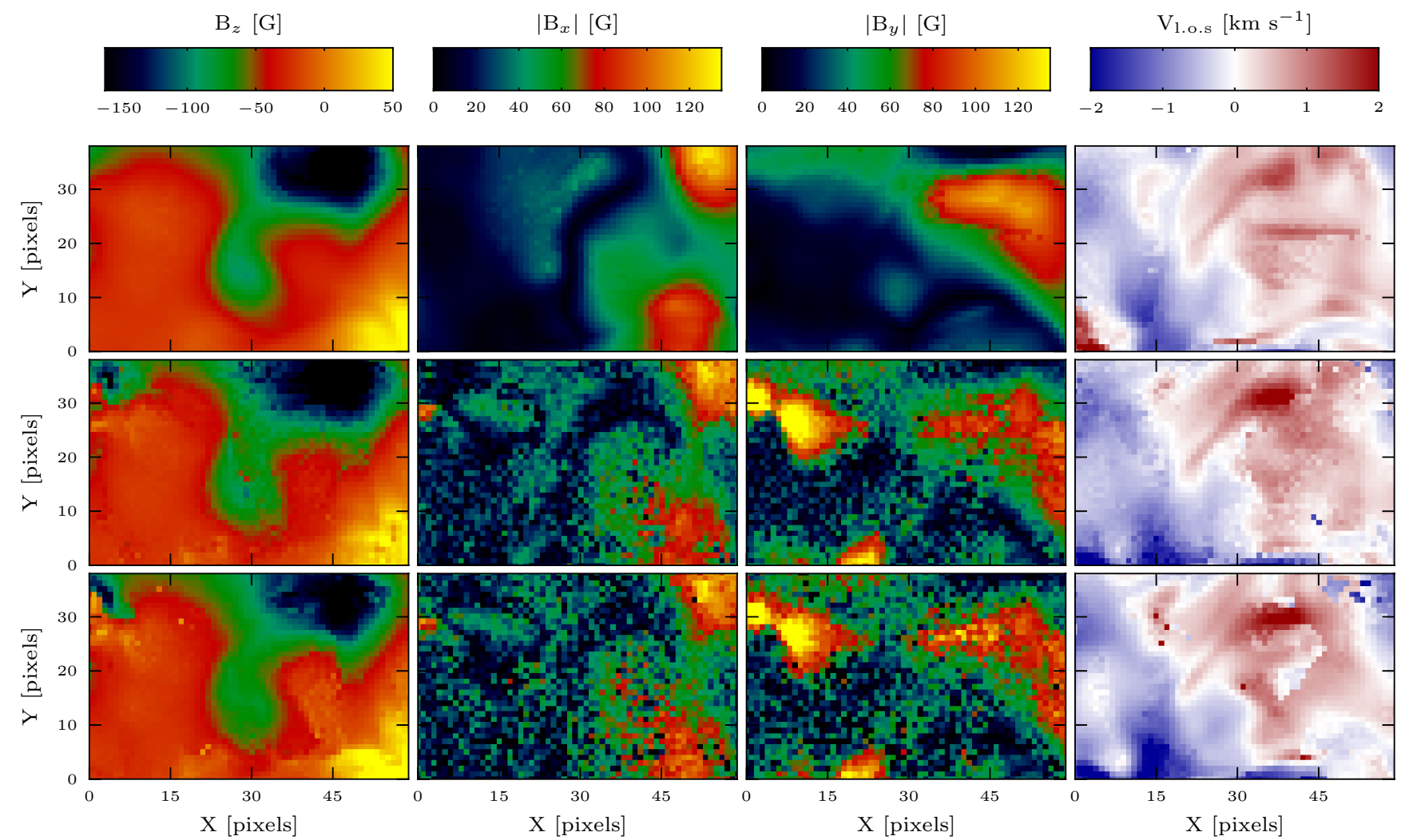

Fig. 14. Results from an inversion carried out in LTE. The top row illustrates quantities from the 3D snapshot, the middle row the results of the NLTE inversion and the bottom row the results of the LTE inversion. In the velocity panel, upflows have positive sign. The inversions were computed assuming a spectral resolution of $50 \mathrm{~m} \AA$ and random noise with $\sigma=10^{-4}$.

correlation coefficients are $r=0.90$ and $r=0.69$ for $B_{x}$ and $B_{y}$, respectively. The lower quality of the inversion compared to the $B_{z}$ case is due to the Stokes $Q$ and $U$ signals being weaker than Stokes $V$. In a realistic case, the data would be dominated by the observational noise, except in specific cases such as strong fields in the vicinity of sunspots.

\section{Discussion and conclusions}

We have computed full Stokes synthetic line profiles using a 3D radiation-MHD simulation of the outer atmosphere of the Sun. A full 3D solution of the NLTE radiative transfer problem was performed using Mutri3D. The width of the core of the synthetic Ca II intensity profiles is approximately a factor 2.5 lower than observed. This would lead to artificially strong Stokes $Q$, $U$ and $V$ signal. To remedy this, external micro-turbulence was added, which brought the synthetic line widths closer to the observed ones.

The resulting line profiles were degraded by various amounts of spectral smearing with various levels of noise added. They were then inverted using the inversion code NicolE, which solves the NLTE radiative transfer problem assuming plane-parallel geometry for each pixel (1.5D). The inversions were performed assuming that the polarization is produced by the Zeeman effect only. This assumption is reasonable for solar regions with moderate to strong activity, which is the most likely target for an application of NICOLE.

We find here that the NLTE inversion can reliably recover the original line-of-sight magnetic field and velocity, with the noise levels and spectral resolutions considered. Temperatures are less reliably inverted. High-temperature areas are well reproduced, but the inversion is less sensitive to cold areas because it cannot properly treat the $3 \mathrm{D}$ effect caused by incoming radiation from hotter neighboring pixels.

The quality of the NLTE inversion for the horizontal field strongly depends on the amount of noise present in the Stokes profiles, and less so on the spectral resolution. Only with a noise level below $\sigma=10^{-3.5}$ is the inversion able to recover the features present in the model atmosphere.

We also tested the reliability of a simpler and faster LTE inversion, which can recover the line-of-sight velocity and the magnetic field, but not the temperature. We showed that this inversion recovers $B_{z}$ and $v_{\text {los }}$ quite well, but yields less reliable results for the horizontal components of the magnetic field. Nevertheless, LTE inversion is a viable and fast strategy if one is interested in the vertical velocity and magnetic field only.

Our results strongly suggest that to be able to detect and invert Stokes $Q$ and $U$ signals in the quiet Sun, observations should have a noise level better than $\sigma=10^{-3.5}$. Instrumental filter widths below $50 \mathrm{~m} \AA$ do not improve the quality of the inversion.

Our work leads to a better understanding of the information contained in chromospheric Ca II observations and what can be learned from their inversions. We showed that a NLTE inversion can reliably recover the vertical magnetic field and velocity at noise levels of $\sigma=10^{-3}$ from a radiation-MHD simulation of quiet Sun, and that the horizontal components can be recovered if the noise level is below $\sigma=10^{-3.5}$. Our results also strongly suggest that if the inversion techniques are applied to observations of more active regions on the Sun, which produce stronger polarization signals, the inferred quantities will be fairly accurate. 
Acknowledgements. H.S.N. gratefully acknowledges financial support by the Spanish Ministry of Science and Innovation through project AYA2010-18029 (Solar Magnetism and Astrophysical Spectropolarimetry) and CONSOLIDER INGENIO CSD2009-00038 (Molecular Astrophysics). This research project has been supported by a Marie Curie Early Stage Research Training Fellowship of the European Community's Sixth Framework Programme under contract number MEST-CT-2005-020395: the USO-SP International School for Solar Physics. This research was supported by the Research Council of Norway through the grant "Solar Atmospheric Modelling" and through grants of computing time from the Programme for Supercomputing. JdlCR gratefully acknowledges financial support by the European Commission through the SOLAIRE Network (MTRN-CT-2006-035484). J.L. acknowledges support from the Netherlands Organization for Scientific Research (NWO).

\section{References}

Asensio Ramos, A., Manso Sainz, R., Martínez González, M. J., et al. 2012, ApJ, 748, 83

Bellot Rubio, L. R., Ruiz Cobo, B., \& Collados, M. 1998, ApJ, 506, 805

Brault, J. W., \& Neckel, H. 1987, Spectral Atlas of Solar Absolute Disk-averaged and Disk-Center Intensity from 3290 to $12510 \AA$, ftp://ftp.hs. uni-hamburg.de/pub/outgolng/FTS-Atlas

Carlin, E. S., Manso Sainz, R., Asensio Ramos, A., \& Trujillo Bueno, J. 2012, ApJ, 751, 5

Carlsson, M. 1986, Uppsala Astronomical Observatory Reports, 33

Cauzzi, G., Reardon, K., Rutten, R. J., Tritschler, A., \& Uitenbroek, H. 2009, A\&A, 503, 577

Charbonneau, P. 1995, ApJS, 101, 309

Fontenla, J. M., Avrett, E., Thuillier, G., \& Harder, J. 2006, ApJ, 639, 441

Hansteen, V. H., Carlsson, M., \& Gudiksen, B. 2007, in The Physics of Chromospheric Plasmas, ed. P. Heinzel, I. Dorotovič, \& R. J. Rutten, ASP Conf. Ser., 368, 107
Hill, G. 1982, Publications of the Dominion Astrophysical Observatory Victoria, 16,67

Keller, C. U., Schüssler, M., Vögler, A., \& Zakharov, V. 2004, ApJ, 607, L59

Lagg, A., Woch, J., Krupp, N., \& Solanki, S. K. 2004, A\&A, 414, 1109

Landi Degl'Innocenti, E. 1992, in Solar Observations: Techniques and Interpretation, First Canary Islands Winter School of Astrophysics, ed. F. Sánchez, M. Collados, \& M. Vázquez (Cambridge Univ. Press), 71

Landi Degl'Innocenti, E., \& Landolfi, M. 2004, Polarization in Spectral Lines, Astrophysics and Space Science Library, 307

Leenaarts, J., \& Carlsson, M. 2009, in ASP Conf. Ser. 415, ed. B. Lites, M. Cheung, T. Magara, J. Mariska, \& K. Reeves, 87

Leenaarts, J., Carlsson, M., Hansteen, V., \& Rouppe van der Voort, L. 2009, ApJ, 694, L128

Leenaarts, J., Rutten, R. J., Reardon, K., Carlsson, M., \& Hansteen, V. 2010, ApJ, 709, 1362

Leenaarts, J., Carlsson, M., \& Rouppe van der Voort, L. 2012, ApJ, 749, 136

Manso Sainz, R., \& Trujillo Bueno, J. 2010, ApJ, 722, 1416

Pietarila, A., Socas-Navarro, H., Bogdan, T., Carlsson, M., \& Stein, R. F. 2006, ApJ, 640, 1142

Pietarila, A., Socas-Navarro, H., \& Bogdan, T. 2007, ApJ, 670, 885

Ruiz Cobo, B., \& del Toro Iniesta, J. C. 1992, ApJ, 398, 375

Rutten, R. J., \& Kostik, R. I. 1982, A\&A, 115, 104

Rutten, R. J., Leenaarts, J., Rouppe van der Voort, L. H. M., et al. 2011, A\&A, 531, A17

Socas-Navarro, H. 2011, A\&A, 529, A37

Socas-Navarro, H., \& Trujillo Bueno, J. 1997, ApJ, 490, 383

Socas-Navarro, H., Trujillo Bueno, J., \& Ruiz Cobo, B. 2000, ApJ, 530, 977

Trujillo Bueno, J. 2010, in Magnetic Coupling between the Interior and Atmosphere of the Sun, ed. S. S. Hasan, \& R. J. Rutten, 118

Trujillo Bueno, J., \& Landi Degl'Innocenti, E. 1996, Sol. Phys., 164, 135

Trujillo Bueno, J., Shchukina, N., \& Asensio Ramos, A. 2004, Nature, 430, 326

Uitenbroek, H. 1989, A\&A, 213, 360

van Noort, M. J., \& Rouppe van der Voort, L. H. M. 2006, ApJ, 648, L67

Wedemeyer-Böhm, S., \& Carlsson, M. 2011, A\&A, 528, A1 